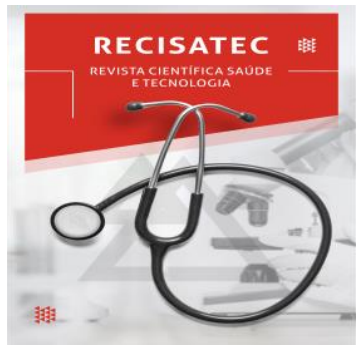

\title{
BIOMEDICINA ESTÉTICA E AS CONTRIBUIÇÕES DO TRATAMENTO CONTRA QUEDA CAPILAR COM PLASMA RICO EM PLAQUETAS: PÓS-COVID-19
}

\author{
AESTHETIC BIOMEDICINE AND THE CONTRIBUTIONS AT THE OF TREATMENT AGAINST \\ CAPILLARY FALL WITH PLATELET RICH PLASMA: POST COVID-19
}

\section{BIOMEDICINA ESTÉTICA Y LAS CONTRIBUCIONES DEL TRATAMIENTO CONTRA LA GOTA CAPILAR CON PLASMA RICO EN PLAQUETAS: POST COVID-19}

\author{
Clecilene Gomes de Carvalho'
}

Submetido em: 09/08/2021

e1213

Aprovado em: 16/09/2021

https://doi.org/10.53612/recisatec.v1i2.13

\section{RESUMO}

A queda capilar pode atingir demasiadamente a autoestima de uma pessoa. A COVID-19, causada pelo Coronavírus (SARS-CoV-2 - Síndrome Respiratória Aguda Grave pode deixar várias sequelas e uma delas é a queda capilar, que pode aparecer mesmo após a recuperação do paciente. Acreditase que a queda é proveniente, sobretudo, devido aos picos febris e pode acometer $1 / 3$ dos pacientes com diagnóstico positivo para a doença. O plasma rico em plaquetas (PRP) é um biomaterial, obtido através do processo de centrifugação, possui fatores de crescimento, como o PDGF, o TGF-beta e o VEGF, que estimularam o crescimento capilar e a sobrevivência de células foliculares. Portanto, levando em consideração que dentre os objetivos do biomédico esteta está o de reestabelecer a autoestima do paciente e que este profissional habilitado pode executar o tratamento com PRP, desde a coleta do material biológico até a realização do procedimento, o objetivo deste trabalho de revisão é pesquisar tanto o papel do biomédico, bem como os benefícios do Plasma rico em plaquetas no tratamento da queda capilar pós a COVID-19.

PALAVRAS-CHAVE: COVID-19. Queda capilar. Plasma rico em plaquetas. Tratamento autólogo. Biomedicina estética.

\begin{abstract}
Hair loss can greatly affect a person's self-esteem. COVID-19, caused by Coronavirus (SARS-CoV-2 Severe Acute Respiratory Syndrome), can leave several sequelae and one of them is capillary loss, which can appear even after the patient's recovery, especially due to fever peaks and can affect $1 / 3$ of patients with a positive diagnosis for the disease. Platelet-rich plasma (PRP) is a biomaterial, after the centrifugation process, it has growth, like PDGF, TGF-beta and VEGF, which stimulate capillary and follicular cell growth. Therefore, taking into account that the goals of the biomedical esthetic are to re-establish the patient's self-esteem and that this qualified professional can perform the treatment with PRP, from the collection of biological material to the performance of the procedure, the objective of this review works to research both the role of the biomedic, as well as the benefits of platelet-rich plasma in the treatment of capillary loss after COVID-19.
\end{abstract}

KEYWORDS: COVID-19. Capillary Fall. Platelet-rich plasma. Autologous treatment. Aesthetic Biomedicine.

\footnotetext{
${ }^{1}$ Graduada em Enfermagem pela UNINCOR. Complementação Pedagógica em Biologia. Graduada em Gestão em Serviços Jurídicos e Notariais pela UNINTER. Graduada em Gerontologia pela UNINTER. Especialização em Enfermagem do Trabalho. Especialização em Saúde Pública com Ênfase em Estratégia Saúde da Família. Especialização em Psicologia da Inteligência Multifocal. Especialização em Estética Facial e Corporal. Especialização em Desenvolvimento e produção de Cosméticos. Especialização em Biomedicina Estética. Especializando em Análises Clínicas e Microbiologia. Especialização em Enfermagem de Urgência e Emergência. Especializando em Abordagem Multidisciplinar em Oncologia. Membro da Associação Mineira de Hipertensão Pulmonar. Graduada em Biomedicina pela UNA. Especializando em Estética Avançada (INAESP/FACOP). E-mail: clecilene@globo.com.
} 


\section{RECISATEC - REVISTA CIENTÍFICA SAÚDE E TECNOLOGIA ISSN 2763-8405}

BIOMEDICINA ESTÉTICA E AS CONTRIBUIÇÕES DO TRATAMENTO CONTRA QUEDA CAPILAR COM PLASMA RICO EM PLAQUETAS: PÓS-COVID-19 Clecilene Gomes de Carvalho

\section{RESUMEN}

La caída del cabello puede afectar en gran medida la autoestima de una persona. EI COVID-19, causado por Coronavirus (SARS-CoV-2 - Síndrome Respiratorio Agudo Severo) puede dejar varias secuelas y una de ellas es la caída del cabello, que puede aparecer incluso después de la recuperación del paciente, principalmente por picos febriles y puede afectar 1 / 3 de los pacientes con diagnóstico positivo de la enfermedad. El plasma rico en plaquetas (PRP) es un biomaterial, obtenido mediante el proceso de centrifugación, posee factores de crecimiento como PDGF, TGF-beta y VEGF, que estimularán el crecimiento del cabello y la supervivencia de las células foliculares. Por ello, se ha sugerido que entre los objetivos de la estética biomédica está la restauración de la autoestima del paciente y que este profesional cualificado pueda realizar el tratamiento con PRP, desde la recogida de material biológico hasta el procedimiento. investigando el papel de la biomedicina y los beneficios del plasma rico en plaquetas en el tratamiento de la caída del cabello después de la COVID-19.

PALABRAS CLAVE: COVID-19. Secuelas capilares. Plasma rico en plaquetas. Tratamiento autólogo. Biomedicina Estética.

\section{INTRODUÇÃO}

Coronavírus são RNAs de vírus causadores de infecções respiratórias, dotados de alta capacidade de mutação e potencial de causar desde um resfriado comum a uma síndrome respiratória fatal (TESINI, 2020; PIEREZAN et al., 2020, CARVALHO; CÉSAR, 2021). O COVID-19 pertence ao subgênero Sarbecovírus da família Coronaviridae e seu modo de transmissão ocorre por contato, por gotículas e aerossol de gotículas respiratórias de pessoas contaminadas e ainda por fômites contaminados (CARVALHO; MAGALHÃES, 2021).

Desde a descoberta da doença várias sequelas foram descobertas em pessoas que contraíram a doença, dentre elas a queda capilar (IZUMI; BRANDÃO, 2021).

A COVID-19, causada pelo Coronavírus (SARS-CoV-2 - Síndrome Respiratória Aguda) pode deixar várias sequelas e uma delas é a queda capilar. A queda de cabelo acentuada pode acometer $1 / 3$ dos pacientes com diagnóstico positivo para a doença (PIEREZAN et al., 2020, IZUMI; BRANDÃO, 2021). Os sintomas podem variar de leves, como: coriza, tosse e dor de garganta até sintomas mais graves como: febre alta, pneumonia e insuficiência respiratória aguda (FIOCRUZ, 2020).

Em um relato de caso realizado por Izume \& Brandão (2021), os pesquisadores concluíram que dentre as principais razões para queda capilar estão os picos febris, que desencadeiam o quadro de eflúvio telógeno temporário.

O Plasma Rico em Plaquetas (PRP) é um biomaterial, obtido através do processo de centrifugação, possui fatores de crescimento, como o PDGF, o TGF-beta e o VEGF, que estimulam o crescimento capilar e a sobrevivência de células foliculares (NASCIMENTO, 2017; FURTADO, DUTRA, 2018). 


\section{RECISATEC - REVISTA CIENTÍFICA SAÚDE E TECNOLOGIA ISSN 2763-8405}

BIOMEDICINA ESTÉTICA E AS CONTRIBUIČ̃ES DO TRATAMENTO CONTRA QUEDA CAPILAR COM PLASMA RICO EM PLAQUETAS: PÓS-COVID-19

A Resolução n 197, de fevereiro de 2011, dispõe sobre as atribuições do profissional biomédico no exercício da saúde estética. Afirma que procedimentos invasivos não cirúrgicos são também de competência dos profissionais da área de saúde, dentre eles o biomédico (CONSELHO FEDERAL DE BIOMEDICINA, 2011). Desta forma, o biomédico esteta habilitado está apto a trabalhar com PRP de forma intradérmica.

Levando em consideração que $43 \%$ a $98 \%$ dos casos têm como sintoma febre $\geq 38,3{ }^{\circ} \mathrm{C}$ (MICHELIN; LINS; FALAVIGNA, 2020) e que $1 / 3$ dos pacientes que contraem a doença são acometidos por queda capilar acentuada (PIEREZAN et al, 2020, IZUMI; BRANDÃO, 2021), e ainda, que dentre os objetivos do biomédico esteta está o de reestabelecer a autoestima do paciente e que este profissional habilitado pode executar o tratamento com PRP, desde da coleta do material biológico até a realização do procedimento, o objetivo deste trabalho de revisão é pesquisar tanto o papel do biomédico, bem como os benefícios do PRP no tratamento da queda capilar pós a COVID19.

\section{METODOLOGIA}

Trata-se de um artigo de revisão integrativa da literatura, que consiste em um método de pesquisa baseado em evidência, na qual o pesquisador reúne, sintetiza e analisa as publicações de um determinado tema com a intenção de solucionar um problema (MENDES; GALVÃO, 2008). O trabalho teve como escolha uma abordagem crítica e de cunho descritivo.

Para compor o corpus deste trabalho foram utilizados artigos pesquisados nas bases de dados do PubMed, Scielo e Google Acadêmico, no período de abril de 2021 a agosto de 2021.

Para a busca, utilizaram-se os seguintes descritores: COVID-19, Plasma Rico em Plaquetas (PRP), queda capilar, papel do biomédico esteta e tratamento autólogo. Quando possível, os descritores foram utilizados de forma associada.

Os critérios de elegibilidade para a pesquisa, relacionados à busca bibliográfica, foram artigos publicados nos idiomas inglês, espanhol e português, publicados nos anos de 2017 a 2021, que tratassem do tema e que, sobretudo, respondessem os objetivos propostos, pesquisas de artigos ou dissertações disponíveis nos meios eletrônicos, e também os que estivessem disponíveis na íntegra. Para utilização de resoluções, não houve data de corte.

De acordo com a Resolução o 510 de 07 de abril de 2016, o presente estudo dispensa avaliação de um Comitê de Ética em Pesquisa de Seres Humanos, pois utiliza informações de domínio público (CONSELHO NACIONAL DE SAÚDE, 2016).

\section{RESULTADOS E DISCUSSÃO}

Dentre as principais queixas no consultório dermatológico, está a queda a capilar que se tornou mais comum durante a pandemia da COVID-19. O principal diagnóstico é o eflúvio telógeno, 


\section{RECISATEC - REVISTA CIENTÍFICA SAÚDE E TECNOLOGIA ISSN 2763-8405}

BIOMEDICINA ESTÉTICA E AS CONTRIBUIÇÕES DO TRATAMENTO CONTRA QUEDA CAPILAR COM PLASMA RICO EM PLAQUETAS: PÓS-COVID-19 Clecilene Gomes de Carvalho

queda capilar mais acentuada na região bitemporal (CASSIANO, 2021; IZUME \& BRANDÃO, 2021). A queda de 100 a 120 fios por dia é considerada normal, porém acima disso, já é considerado patológico (SBD, 2021).

O fio tem três fases, anágena _ caracterizada por intensa atividade mitótica na matriz do folículo piloso, fio em crescimento, cresce $01 \mathrm{~cm}$ por mês e o processo permanece ao longo de três anos; catágena _ fio maduro ainda no folículo e tem duração de duas a três semanas, fase em que a atividade celular é reduzida e por isso o bulbo entra em processo de atrofia; telógena _ o pelo se separa da papila dérmica, fio em queda já destacado do folículo, pode durar até quatro meses. A maioria dos fios está em fase de crescimento (NASCIMENTO, 2017; CASSIANO, 2021; ESTADO DE MINAS, 2021).

“Em indivíduos normais, 83\% a 90\% das hastes estão em fase anágena. Entre 11\% e 15\% apresentam-se em fase telógena e apenas 1\% ou menos na fase catágena" (NASCIMENTO, 2017).

O Eflúvio telógeno é o aumento da queda capilar diária que acontece devido à antecipação do ciclo de vida do cabelo, o fio muda da fase de crescimento para fase de queda, e é dividido em agudo e crônico (BERGFELD, 2020). O agudo é caracterizado por eventos que aconteceram três meses antes da queda capilar.

Pode acontecer devido pós-parto, febre, infecção aguda, sinusite, pneumonia, gripe, dietas muito restritivas, doenças metabólicas ou infecciosas, cirurgias, especialmente a bariátrica, por conta da perda de sangue e do estresse metabólico, além do estresse (SBD, 2021).

A fase crônica é tida como uma queda difusa de cabelo, com duração superior a seis meses (IZUME; BRANDÃO, 2021). Esta fase ocorre de forma cíclica, pode ocorrer duas vezes por ano ou até mesmo a cada dois anos (SBD, 2021).

Em relação à queda após a COVID-19, os fios começam a cair em volume bem maior do que o normal, de forma aguda, em torno de dois ou três meses depois da infecção (ESTADO DE MINAS, 2021).

Dentre os tratamentos contra queda capilar está o uso do PRP, derivado de sangue autólogo, assim, não existe contraindicação, não há risco de rejeição ou processo alérgico (NASCIMENTO, 2017).

O PRP aumenta vascularização local; Aumenta aporte de oxigênio e nutrição na pele; Induz a cicatrização por primeira intenção; Libera fatores de crescimento; Gera novos folículos, além de tratar: Rugas; Linhas de expressão; Sulcos; Poros dilatados; Estrias; Alopecia; Flacidez de pele (incluindo pós bariátrico e lipoaspiração); Cicatriz atrófica como acne, previne quelóide e hipertrófica (ANVISA, 2018).

O PRP é constituído basicamente por três componentes, o plasma, leucócitos e plaquetas (NASCIMENTO, 2017).

Para a realização deste procedimento é necessário a venopunção. A coleta é feita em tubo Azul contendo citrato de sódio que interrompe cascata de coagulação. Em um segundo momento o 


\section{RECISATEC - REVISTA CIENTÍFICA SAÚDE E TECNOLOGIA ISSN 2763-8405}

BIOMEDICINA ESTÉTICA E AS CONTRIBUIC̄õES DO TRATAMENTO CONTRA QUEDA CAPILAR COM PLASMA RICO EM PLAQUETAS: PÓS-COVID-19

sangue é centrifugado, onde ocorrerá à separação dos três componentes. O PRP é rico em fatores de crescimento como o PDGF - fator de crescimento derivado de plaquetas, TGF-beta _ Fatores de crescimento de transformação beta, EGF _ Fator de crescimento da epiderme. Os fatores de crescimento como o endotélio vascular (VEGF), o fator de crescimento epidérmico (EGF) e o fator de crescimento de fibroblastos (FGF) ajudam na criação do folículo piloso, pois ativam sua função e promovem o crescimento do cabelo (NASCIMENTO, 2017).

Considerando a Resolução nำ 197, de 21 de fevereiro de 2011, que define as atribuições do Biomédico quanto ao exercício na área de saúde estética e dentre elas a realização de procedimentos invasivos não cirúrgicos, como é o caso PRP via intradérmica, este profissional está apto a realizar o tratamento (CONSELHO FEDERAL DE BIOMEDICINA, 2011).

O uso do PRP pós COVID-19 deve ser iniciado o quanto antes, visto que o PRP estimula a proliferação de células da camada dermopapilar, o que aumenta a sobrevivência de células do folículo piloso através de seus efeitos antiapoptóticos possivelmente estimulando o crescimento capilar, e ainda prolonga a fase anágena. O PDGF induz a proliferação de células-tronco no bulbo folicular, o TGF-beta estimula as células da papila dérmica, o que poderia evitar maiores danos ao paciente no eflúvio telógeino, sobretudo devido aos vários fatores de crescimento contidos do PRP (FURTADO, DUTRA, 2021).

\section{CONSIDERAÇÕES FINAIS}

O ser humano é afetado emocionalmente quando sua estética está comprometida, o que o leva a diminuição da autoestima.

O eflúvio telógeno, queda capilar pós COVID-19, trata-se de um quadro temporário, causado pelos picos febris, mas que pode ser abrandado com o uso do PRP, antes do início abrupto da queda capilar, graças aos fatores de crescimento contidos no PRP.

O PRP é um biomaterial, obtido através do processo de centrifugação do sangue coletado do paciente pelo biomédico esteta habilitado.

Os fatores de crescimento contidos no PRP como o VEGF - fatores de crescimento como o endotélio vascular, o fator de crescimento epidérmico (EGF) e o fator de crescimento de fibroblastos (FGF) ajudam na criação do folículo piloso, pois ativam sua função e promovem o crescimento do cabelo, aumentam a vascularização local e o aporte de oxigênio, nutrindo e favorecendo a geração de novos folículos

Assim, o biomédico habilitado, tendo um olhar holístico capaz de avaliar adequadamente o quadro, terá um papel importante, tanto na produção do PRP, quando na sua aplicação correta.

Contudo, vale ressaltar que os estudos devem ser constantes, pois lidar com algo novo, como é o caso da COVID-19, exige pesquisas exaustivas, pois, assim como o vírus se mostra altamente mutante, a necessidade de aprofundar o conhecimento, no que tange esta doença e suas complicações é primordial. 


\section{RECISATEC - REVISTA CIENTÍFICA SAÚDE E TECNOLOGIA ISSN 2763-8405}

BIOMEDICINA ESTÉTICA E AS CONTRIBUIČ̃ES DO TRATAMENTO CONTRA QUEDA CAPILAR COM PLASMA RICO EM PLAQUETAS: PÓS-COVID-19 Clecilene Gomes de Carvalho

\section{REFERÊNCIAS}

ANVISA. Agência Nacional de Vigilância Sanitária. Evento discutiu regulação de plasma rico em plaquetas. Brasília: Anvisa, 2018. Disponível em: https://www.gov.br/anvisa/pt-br/assuntos/noticiasanvisa/2018/evento-discutiu-regulacao-de-plasma-rico-emplaquetas\#: :text=Para\%20promover\%200\%20debater\%20sobre,audit\%C3\%B3rio\%20da\%20Ag\%C 3\%AAncia\%2C\%20em\%20Bras\%C3\%ADlia. Acesso em: 04 abr. 2021.

BERGFELD, Wilma. Telogen effluvium. UpToDate, 2020. Disponível em: https://www.uptodate.com/contents/telogen-effluvium. Acesso em: 13 ago. 2021.

BRASIL. Ministério da Saúde. Secretaria de Vigilância em Saúde. Departamento de Análise em Saúde e Doenças não Transmissíveis. Guia de vigilância epidemiológica Emergência de saúde pública de Importância nacional pela Doença pelo coronavírus 2019 -covid-19 [recurso eletrônico]. Brasília: Ministério da Saúde, 2021.

CARVALHO, Clecilene G.; CÉSAR, Jorgino J. A importância da notificação compulsória: COVID-19. Braz. J. Surg. Clin. Res, v. 34, n. 1, p.11-16, Paraná, 2021. Disponível em: https://www.mastereditora.com.br/bjscr34-. Acesso em: 16 maio 2021.

CARVALHO, Clecilene G.; MAGALHÃES, Sérgio R. COVID 19: uma emergência de saúde públicaalguns aspectos da introdução da vacinação em Contage rm (MG). Recima21-Revista Cientifica Multidisciplinar, São Paulo, v. 2 n. 5, 2021. Disponível em: https://recima21.com.br/index.php/recima21/issue/view/9. Acesso em: 16 maio 2021.

CASSIANO, Daniel. Queda de cabelo pode estar associada à pandemia de Covid-19. Veja saúde, São Paulo, 2021. Disponível em: https://saude.abril.com.br/blog/com-a-palavra/queda-de-cabelopode-estar-associada-a-pandemia-de-covid-19/. Acesso em 22 junho de 2021.

CONSELHO NACIONAL DE SAÚDE. Resolução 510 de 07 de abril de 2016. Brasília: Conselho Nacional de Saúde, 2016. Disponível em: http://conselho.saude.gov.br/resolucoes/2016/Reso510.pdf. Acesso em: 08 ago 2021.

CONSELHO FEDERAL DE BIOMEDICINA. Dispõe sobre as atribuições do profissional Biomédico no Exercício da Saúde Estética e Atuar como Responsável Técnico de Empresa que Executam Atividades para fins Estéticos. Resolução no 197, de 21 de fevereiro de 2011. São Paulo: CFB, 2011. Disponível em: http://cfbm.gov.br/wp-content/uploads/2016/06/Res-2011197.pdf. Acesso em: 02 jul. 2021.

ESTADO DE MINAS - Sessão Internacional. Efeitos da Covid-19: o que causa e como tratar a queda de cabelo que afeta 1 em 4 infectados. Belo Horizonte, 2021. Disponível em: https://www.em.com.br/app/noticia/internacional/bbc/2021/03/15/interna internacional,1246700/efeito s-da-covid-19-o-que-causa-e-como-tratar-a-queda-de-cabelo-que-afeta.shtml. Acesso em: 10 ago. 2021.

FIOCRUZ - Fundação Oswaldo Cruz. Ministério da Saúde. COVID 19 - Novo coronavírus. Brasília: FIOCRUZ, 2020. Disponível em:

http://www.iff.fiocruz.br/pdf/Informativos coronavirus 2\%20SEM\%20NEO.pdf. Acesso em: 16 maio 2021.

FURTADO, Luana V.; DUTRA, Robertson T. Tratamento da alopecia androgenética através da aplicação de plasma rico em plaquetas: uma revisão de literatura. Biociencias, biotecnologia e saúde, Paraná, v. 11, n. 21, 2018. 


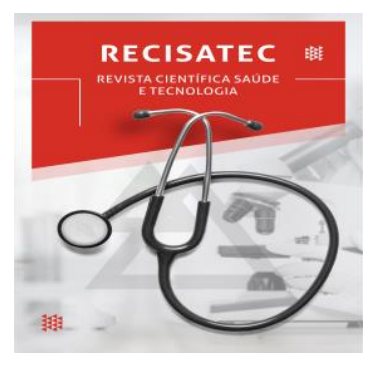

IZUMI, Marcella O.; BRANDÃO, Byron J. F. Tratamento do Eflúvio Telógeno Pós-Covid 19. BWS Journal, São Paulo, v. 4, 2021. Disponível em:

https://bwsjournal.emnuvens.com.br/bwsi/article/view/165. Acesso em: 16 jul. 2021.

MENDES, K. D. S.; SILVEIRA, R. C. C. P.; GALVÃO, C. M. Revisão integrativa: método de pesquisa para a incorporação de evidências na saúde e na enfermagem. Texto \& Contexto-Enfermagem, v. 17, n. 4, p. 758-764, 2008.

MICHELIN, Lessandra; LINS, Rodrigo S.; FALAVIGNA, Asdrubal. COVID-19: perguntas e respostas Centro de Telemedicina da UCS. Caxias do Sul: EDUCS, 2020. Disponível em:

https://www.ucs.br/site/midia/arquivos/ebook-covid19-editora.pdf. Acesso em: 08 ago. 2021.

NASCIMENTO, Fabianne B. Utilização do plasma rico em plaquetas no tratamento da alopecia androgenética masculina: uma revisão de literatura. 2017. TCC (Monografia) - Instituto Nacional de Ensino Superior e Pesquisa e Centro de Capacitação Educacional, Recife, 2017. Disponível em: https://www.ccecursos.com.br/img/resumos/biomedicina-estetica/tcc---fabiana-borges-donascimento.pdf. Acesso em 05: ago. 2021.

PIEREZAN, Allana C.; et al. Consequências do novo coronavírus: queda capilar, um feito secundário do COVID-19? In.: Anais... XXV Seminário Internacional de Ensino, Pesquisa e Extensão Desafios da Ciência em tempos de pandemia. Rio Grande do Sul, 2020. Disponível em: https://home.unicruz.edu.br/seminario/exposicao/posteres/iniciacao saude/ID 431.pdf. Acesso em: 16 abr. 2021.

SBD - Sociedade Brasileira de Dermatologia. Eflúvio Telógeno. Rio de Janeiro: SBD, 2021. Disponível em: https://www.sbd.org.br/dermatologia/cabelo/doencas-e-problemas/efluviotelogeno/56/. Acesso em: 13 ago. 2021.

TESINI, B. L. University of Rochester School of Medicine and Dentistry. Coronavírus e síndromes respiratórias agudas (covid-19, mers e sars). Manual MDS -Versão para profissionais de saúde, abril de 2020. Disponível em: https://www.msdmanuals.com/pt/profissional/doen\%C3\%A7asinfecciosas/v\%C3\%ADrus-respirat\%C3\%B3rios/coronav\%C3\%ADrus-e-s\%C3\%ADndromesrespirat\%C3\%B3rias-agudas-covid-19,-mers-e-sars. Acesso em: 14 ago. 2021. 